\title{
Responsiveness and minimal important change for the quick-DASH in patients with shoulder disorders
}

\author{
Cecilie Rud Budtz ${ }^{1 *}$ (D) Johan Hviid Andersen', Nils-Bo de Vos Andersen² and David Høyrup Christiansen ${ }^{1,3}$
}

\begin{abstract}
Background: Responsiveness and minimal important change (MIC) are central measurement properties when interpreting scores from health questionnaires. The aim of the study was to evaluate the responsiveness and MIC of the Danish version of the shortened version the Disabilities of the Arm, Shoulder and Hand questionnaire (Quick-DASH) in patients with shoulder disorders referred to primary care physiotherapy treatment.

Methods: The study included 261 patients who completed questionnaires at baseline and 3 and 6 months follow up. Absolute and relative change scores was analysed using receiver-operating-characteristics (ROC) curve analysis with the Patient Global Impression of Change (PGIC) as external anchor.

Results: At both 3 and 6 months follow up, the Area under the Curve (ROC AUC) exceeded 0.70 and MIC was 9.1 and 13.6 at 3 and 6 months respectively.

Conclusion: The Danish version of the Quick-DASH demonstrated adequate ability to measure changes in disability over 3 and 6 months in patients with shoulder disorders undergoing primary care physiotherapy treatment.
\end{abstract}

Keywords: Outcome measure, Responsiveness, Quick-DASH

\section{Background}

Reliable, valid and responsive outcome measures are important when evaluating the effect of treatment. The Disabilities of the Arm, Shoulder and Hand questionnaire (DASH) is a 30 item questionnaire designed to measure symptoms and physical functioning in patients with any or multiple musculoskeletal symptoms of the upper extremity [1]. A major advantage of this questionnaire is that it can be used for any upper extremity evaluation meaning versatility for clinicians and researchers [2]. The DASH has previously been cross cultural adapted to Danish and has been found reliable, valid and responsive among orthopaedic patients with various hand and shoulder diagnoses [3, 4]. However, it is important that a questionnaire is short and easy to complete thereby minimizing the burden on the respondent and limiting missing data. Accordingly, a

\footnotetext{
* Correspondence: cecbud@rm.dk

'Department of Occupational Medicine, Regional Hospital West Jutland,

University Research Clinic, GI. Landevej 61, 7400 Herning, Denmark

Full list of author information is available at the end of the article
}

shortened version of the DASH has been developed (Quick-DASH) [2]. Although such short form questionnaires seem as an attractive and sensible choice, it is essential to insure that measurement properties are maintained [2]. This also applies to the Danish version of the Quick-DASH, which only recently has been evaluated with respect to reliability and construct validity in patients with wrist fractures [5] or total wrist arthroplasty [6].

A central measurement property of scales to assess treatment outcomes is responsiveness. Responsiveness is defined as the ability of a questionnaire to detect change over time in the domain of interest, and it includes the Minimal Important Change (MIC) which is the smallest change in score that would likely be important from the patient's perspective [7]. To ensure the MIC can be distinguished from measurement error, the MIC should ideally exceed the Minimal Detectable Change (MDC) representing the smallest within-person change in score that can be interpreted as real change beyond measurement error. Responsiveness has been evaluated for

(c) The Author(s). 2018 Open Access This article is distributed under the terms of the Creative Commons Attribution 4.0 International License (http://creativecommons.org/licenses/by/4.0/), which permits unrestricted use, distribution, and reproduction in any medium, provided you give appropriate credit to the original author(s) and the source, provide a link to the Creative Commons license, and indicate if changes were made. The Creative Commons Public Domain Dedication waiver (http://creativecommons.org/publicdomain/zero/1.0/) applies to the data made available in this article, unless otherwise stated. 
Quick-DASH in other countries, various populations and settings with divergent results $[8,9]$. Responsiveness of the Danish version of the Quick-DASH has however never been evaluated using the recommended anchorbased method or in a population of shoulder patients. As responsiveness and MIC are likely to depend on population and contextual characteristics, evaluation should be conducted within the setting in which the questionnaire is going to be utilised [10]. The aim of the present study was to evaluate responsiveness and MIC of the Danish Quick-DASH in patients with shoulder disorders referred to primary care physiotherapy treatment, thereby adding to earlier findings on the use of this questionnaire. We hypothesised that the QuickDASH would 1) demonstrate adequate ability to discriminate between improved and unchanged patients and 2) MIC thresholds would exceed the minimal detectable change.

\section{Methods}

\section{Design and population}

The study was nested in a larger prospective cohort study of patients seeking treatment for neck, shoulder, or low-back pain in 23 physiotherapy practices across Denmark from January to June 2016 [11]. Consecutive patients were invited to participate if they were aged 18 years or above and able to understand Danish well enough to self-complete the questionnaires. For the current study patients presenting with shoulder pain were included.

\section{Data collection}

Questionnaire and clinical data were collected using an existing web-based clinical database (www.fysdb.dk). Patients who agreed to participate in the study were asked to complete online questionnaires 1-2 days prior to the first physiotherapy consultation (baseline) and at 3 and 6 months follow up. Participants were notified by e-mail when the follow-up questionnaires were available for completion. The questionnaires included information on gender, age, education level, pain (scale 0-10) and disability (Quick-DASH).

\section{Quick-DASH and patient global impression of change scale}

The Quick-DASH questionnaire contains 11 items (scored on a 0-5 Likert scale) that measure upper limb physical disability and symptoms. Each item has 5 response options from 1 (no difficulty to perform, no symptom or no impact) to 5 (unable to do, very severe symptom or high impact). The responses are summed to a raw score and converted to a 0 (no disability) to 100 (most severe disability) score using the following formula [(sum of score $/ n)-1] \times 25, n$ being the number of completed responses [2].

The Patient Global Impression of Change (PGIC) at 3 months formed the external anchor. Patients were asked to rank their overall state in relation to time of referral on a 7 point scale from much better to much worse. The PGIC scale is widely used in clinical research as a way to quantify the patient's perception of improvement over time [12].

\section{Measurement error of the quick-DASH}

The measurement error of the Quick-DASH has never been established in a population of Danish primary care patients with shoulder disorders. Reliability was consequently established based in a subsidiary sample of 30 consecutive shoulder patients (mean (SD) age 49 [12]; 18 women)). The patients filled in the Quick-DASH twice with a median of 5 days [IQR 3;7] between administrations. The intraclass correlation coefficient (ICC) was 0.87 (95\% CI: 0.79; 0.96)) and the Standard Error of Measurement (SEM) was estimated to 6.0 (95\% CI: 4.7; 8.0), which yielded a Minimal Detectable Change (MDC) of 16.5 (95\% CI 13.1; 22.2) [10].

\section{Statistical analyses}

Descriptive statistics were calculated and presented. Responsiveness was evaluated using an external anchor approach as recommended in the COSMIN guidelines [3]. Receiver-operating-characteristics (ROC) curve analysis of the absolute and relative change scores in the Quick-DASH was used to assess the measurements ability to correctly classify patients as improved (much better or better) or unchanged (little better, unchanged or little worse) according to the PGIC scale. As only few patients ( $n=3$ at 3 months, $n=5$ at 6 months) were worse or much worse, responsiveness to worsening was not analysed. In ROC curve analysis, sensitivity and 1-specificity are plotted at several cut-off points, and the area under the curve (ROC AUC) can be estimated. An ROC AUC greater than 0.70 is considered adequate [10]. All absolute and relative changes and ROC AUC were calculated with $95 \%$ CI. Minimal important change (MIC), estimated using the cut off point at the ROC curve closest to the upper left-hand corner and where sensitivity and specificity was most balanced, represents the score that best discriminates between improved and unchanged patients (ROC MIC). Furthermore, we calculated the 95\% limit cut off point (MIC 95\% upper limit), defined as the upper limit of the distribution of patients who were not importantly changed according to the external anchor (mean change $+1.645 \mathrm{x}$ SD change of the unchanged group) [13]. Missing items in the Quick DASH were handled according to recommendations meaning if only 1 item was missing, the score was 
divided by 10 instead of 11 and if more than 1 item were missing the total score was not calculated [2]. All statistical analyses were performed using STATA Version 15 (StataCorp LP, College Station, TX).

\section{Results}

A total of 341 patients with shoulder conditions were referred from general practitioner to physiotherapy treatment from January to June 2016 of which 5 were excluded (age under $18(n=4)$ and suspicion on serious disease $(n=1))$. A total of 336 patients were invited to participate in the study, 30 patient declined to participate and 45 patients had more than 1 item missing in their baseline Quick-DASH questionnaire, resulting in 261 patients included at baseline (78\%). A total of 73 patients at 3 months and 85 patients at 6 months were lost to follow up, resulting in 188 patients (72\%) at 3 months and 176 patients (67\%) at 6 months to be included in the analyses. Baseline characteristics of included patients $(n=261)$ are presented in Table 1 .

No differences in baseline variables between responders and non-responders at 3 and 6 months were detected (data not shown).

At 3 and 6 months a total of 129 and 125 patients were improved and 59 and 51 were unchanged respectively. Table 2 present mean and relative change scores, ROC curve statistics and ROC MIC values at 3 and 6 months. The absolute ROC AUC was $0.84(0.79 ; 0.90)$ at 3 months and $0.83(0.77 ; 0.90)$ at 6 months. At 3 months the ROC MIC was 9.1 points (sensitivity: $73.6 \%$; specificity: $74.5 \%$; correctly classified: $73.9 \%$ ) and at 6 months the ROC MIC was 13.6 points (sensitivity: 75.2\%;

Table 1 Baseline characteristics $(n=261)$

\begin{tabular}{ll}
\hline Variable & Value* $^{*}$ \\
\hline Sex & $139(53)$ \\
Male & $122(47)$ \\
Age, mean (SD) & $52(14)$ \\
Occupational status & \\
Employed & $179(69)$ \\
Unemployed & $6(2)$ \\
Retired/early retirement/flex job/disability pension & $58(22)$ \\
Student/on leave & $14(5)$ \\
Missing & $4(2)$ \\
Sick leave because of shoulder symptoms & $24(9)$ \\
QuickDASH 0-100, mean (SD) & $35.1(18.4)$ \\
Pain 0-10, mean (SD) & $5.7(2.1)$ \\
\hline Abbrevatons SD standard &
\end{tabular}

Abbreviations: SD standard deviation, Quick-DASH, shortened version of the Disabilities of the Arm, Hand and Shoulder

*Values are $\mathrm{n}(\%)$ unless stated otherwise
Table 2 Mean change scores, ROC curve statistics and MIC values for the Quick-DASH at 3 and 6 months*

\begin{tabular}{lll}
\hline & 3 months & 6 months \\
\hline Absolute mean changes & & \\
Improved & $21.4(18.4 ; 24.3)$ & $24.9(21.8 ; 28.0)$ \\
Unchanged & $1.8(-1.27 ; 4.91)$ & $4.9(1.0 ; 8.8)$ \\
Relative mean changes, \% & & \\
Improved & $58.1(52.3 ; 63.8)$ & $68.7(63.8 ; 73.7)$ \\
Unchanged & $-3.3(-19.1 ; 12.6)$ & $6.8(-8.8 ; 22.3)$ \\
Absolute ROC AUC & $0.84(0.79 ; 0.90)$ & $0.83(0.77 ; 0.90)$ \\
Relative ROC AUC & $0.86(0.81 ; 0.92)$ & $0.90(0.85 ; 0.95)$ \\
MIC values & & \\
MIC ROC ${ }^{\text {a }}$ & 9.1 & 13.6 \\
MIC 95\% upper limit ${ }^{\text {b }}$ & 21.3 & 27.8 \\
MIC percentages $^{c}$ & 33.3 & 41.7 \\
\hline
\end{tabular}

Abbreviations: AUC Area Under the Curve, MIC Minimal important change, $R O C$ Receiver operating characteristics, Quick-DASH, shortened version of the Disabilities of the Arm, Hand and Shoulder

*Values in parentheses are $95 \%$ confidence interval. 3 months: improved $(n=$ 129); unchanged ( $n=59), 6$ months: improved $(n=125)$; unchanged $(n=51)$

a Estimated as the optimal cutoff point of the ROC curve using absolute change scores

${ }^{\mathrm{b}}$ Estimated as the $95 \%$ cutoff limit for the unchanged group

' Estimated as the optimal cutoff point of the ROC curve using relative change scores

specificity: 76.5\%; correctly classified: 75.6\%). Figure 1 presents the ROC curve at 3 and 6 months.

\section{Discussion}

The Danish version of the Quick-DASH demonstrated adequate responsiveness among physiotherapy shoulder patients treated in primary care. At both 3 and 6 months follow up, the lower limit of the 95\% CI of ROC AUC estimates exceeded 0.70, suggesting that the questionnaires ability to correctly classify patients as improved or unchanged was adequate. The estimated ROC MIC at 3 and 6 months was 9.1 and 13.6 points respectably and thereby did not exceed the Minimal Detectable Change (MDC) threshold of 16.5 points established for the present study.

The large cohort of consecutive patients with shoulder disorders are likely to be representative of shoulder patients seen in primary care physiotherapy. The patients had relatively high pain scores and modest disability scores at baseline, which is similar to findings from other Danish cohorts of primary care musculoskeletal physiotherapy patients $[14,15]$. A limitation of the study was the participation rate at baseline $(78 \%)$ and modest follow-up rates at 3 and 6 months (72 and 67\% respectively). This may have affected the generalizability of our findings and we cannot preclude attrition bias, although no differences in baseline variables between responders and non-responders at 3 and 6 months were detected. Also 45 patients were excluded at baseline as they had 

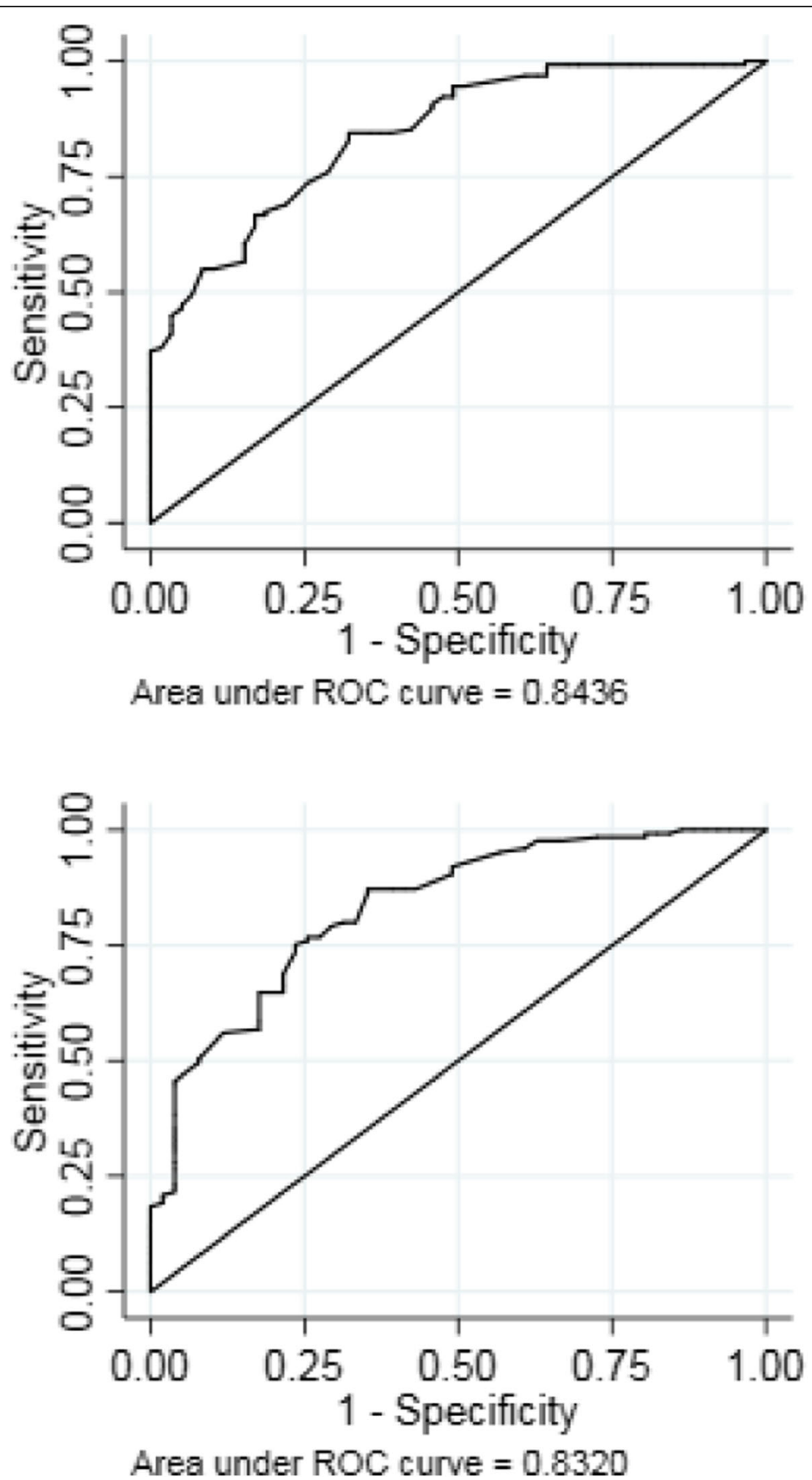

Fig. 1 Receiver-Operating-Characteristics (ROC) curve for the Quick-DASH at 3 months (upper) and 6 months (lower) follow up. The point nearest the upper left hand corner represents the minimal important change (MIC ROC)

more than one item missing in the QuickDASH, indicating the questionnaire may have problems with content validity. Furthermore, the use of transitional scales, such as the PGIC scale, as external anchor has been questioned, as it may be subject to recall bias $[7,16]$. However, it remains the only option as no gold standard exists for measuring subjective changes in disability over time $[12,16]$. The test-retest reliability was based on a separate but similar population and it cannot be precluded that the SEM and MDC would have differed if the test-retest had been performed in a subset of the large cohort. Also the test-retest was performed on 30 patients, which only meets a fair rating in the COSMIN rating of the methodological quality [17]. The results 
were however in line with previously reported ICC values which was evaluated in 30 and 22 patients [13, $14]$, and the results are therefore considered reliable.

Only a few studies have examined responsiveness and MIC of the Quick-DASH in patients undergoing physiotherapy treatment for shoulder disorders [17-20]. Our findings on ROC AUC coincides with 3 studies evaluating responsiveness using PGIC as external anchor, with a ROC AUC ranging from 0.82 to 0.86 [17-19]. There is considerable discrepancies in the reported MIC values in these previous studies, as they range from 8 to 19 [17, $18,20]$. These findings are all based on follow-up periods of 4-6 weeks, and to our knowledge no other study has examined MIC over a longer follow-up period in a similar population. Interestingly, our findings indicate that MIC is not stable over time, as it increases from 9.1 to 13.6 points. The MDC of 16.5 points represent the smallest within-person change in score that can be interpreted as real change beyond measurement error. In contrast to previous studies [13, 16] MIC did not exceed the MDC in the present study. However, it should be noted that these previous studies calculated the MDC with a $90 \%$ confidence level $\left(\mathrm{MDC}^{90}\right)$ (11-13 points), whereas we calculated the $95 \%$ confidence level $\left(\mathrm{MDC}^{95}\right)$. The estimated $\mathrm{MDC}^{90}$ in our study would be 13.9 points, and thereby not far from these previous reported $[20,21]$ results and almost equal to our estimated MIC at 6 months. When interpreting individual absolute change in patients in clinical practise and research the MIC 95\% upper limit may be a preferable choice, on the other hand, as MIC estimates are likely to be influenced by baseline scores [22], MIC percentages may be an even better choice. Our findings on relative MIC scores of 33 and $41 \%$ at 3 and 6 months coincide with common thresholds of clinically relevant important change of > $30 \%$ improvement from baseline to follow-up [21].

In addition, the findings that both absolute and relative minimally important change scores vary over time calls for further investigation into variations over time.

\section{Conclusion}

The Danish version of the Quick-DASH demonstrated adequate ability to measure changes in disability over 3 and 6 months in patients with shoulder disorders undergoing primary care physiotherapy treatment. Minimal important change values in ROC estimates were 9.1 and 13.6 points at 3 and 6 months respectively and did not exceed the $\mathrm{MDC}_{95}$ value in the present study. To insure that individual change scores will exceed measurement error, the MIC 95\% upper limit of 21 and 28 or the relative change scores of $33 \%$ or $41 \%$ may be more preferable when interpreting clinical importance of individual changes in Quick-DASH score over time.

\section{Abbreviations}

AUC: Area Under the Curve; Cl: Confidence Interval; MDC: Minimal

Detectable Change; MIC: Minimal Important Change; PGIC: Patient Global Impression of Change; Quick-DASH: Shortened version of the Disabilities of the Arm, Shoulder and Hand questionnaire; ROC: Receiver-OperatingCharacteristics; SD: Standard Deviation; SEM: Standard Error of Measurement

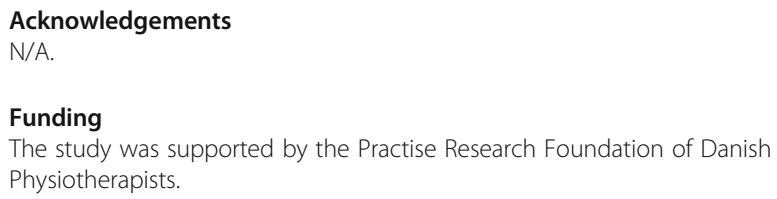

\section{Author's information}

N/A.

\section{Ethics approval and consent to participate}

All participants signed written informed consent forms and the study was approved by the Danish Data Protection Agency (No. 2012-58-006). Under Danish law this study did not need ethics approval (Act on Research Ethics Review of Health Research Projects, October 2013) [23]

\section{Consent for publication}

N/A.

\section{Competing interests}

The authors declare that they have no competing interests.

\section{Publisher's Note}

Springer Nature remains neutral with regard to jurisdictional claims in published maps and institutional affiliations.

\section{Author details}

${ }^{1}$ Department of Occupational Medicine, Regional Hospital West Jutland, University Research Clinic, Gl. Landevej 61, 7400 Herning, Denmark. ${ }^{2}$ Central Denmark Region, Primary Health Care and Quality Improvement, Skottenborg 26, 8800 Viborg, Denmark. ${ }^{3}$ Department of Clinical Medicine, Health, Aarhus University, Palle Juul-Jensens Boulevard 82, 8200 Aarhus, Denmark.

Received: 4 September 2018 Accepted: 27 November 2018 Published online: 10 December 2018

\section{References}

1. Hudak PL, Amadio PC, Bombardier C. Development of an upper extremity outcome measure: the DASH (disabilities of the arm, shoulder and hand) [corrected]. The upper extremity collaborative group (UECG). Am J Ind Med. 1996;29(6):602-8.

2. Beaton DE, Wright JG, Katz JN, Upper Extremity Collaborative Group. Development of the QuickDASH: comparison of three item-reduction approaches. J Bone Joint Surg Am. 2005;87(5):1038-46.

3. Herup A, Merser S, Boeckstyns M. Validation of questionnaire for conditions of the upper extremity. Ugeskr Laeger. 2010;172(48):3333-6.

4. Lundquist CB, Dossing K, Christiansen DH. Responsiveness of a Danish version of the disabilities of the arm, shoulder and hand (DASH) questionnaire. Dan Med J. 2014;61(4):A4813.

5. Schonnemann JO, Eggers J. Validation of the Danish version of the quickdisabilities of arm, shoulder and hand questionnaire. Dan Med J. 2016; 63(12):A5306. 
6. Boeckstyns ME, Merser S. Psychometric properties of two questionnaires in the context of total wrist arthroplasty. Dan Med J. 2014;61(11):A4939.

7. Revicki D, Hays RD, Cella D, Sloan J. Recommended methods for determining responsiveness and minimally important differences for patient-reported outcomes. J Clin Epidemiol. 2008;61(2):102-9.

8. Kennedy CA, Beaton DE, Smith P, Van Eerd D, Tang K, Inrig T, et al. Measurement properties of the QuickDASH (disabilities of the arm, shoulder and hand) outcome measure and cross-cultural adaptations of the QuickDASH: a systematic review. Qual Life Res. 2013;22(9):2509-47.

9. St-Pierre C, Desmeules F, Dionne CE, Fremont P, MacDermid JC, Roy JS. Psychometric properties of self-reported questionnaires for the evaluation of symptoms and functional limitations in individuals with rotator cuff disorders: a systematic review. Disabil Rehabil. 2016;38(2):103-22.

10. Terwee $C B$, Bot SD, de Boer MR, van der Windt DA, Knol DL, Dekker J, et al. Quality criteria were proposed for measurement properties of health status questionnaires. J Clin Epidemiol. 2007;60(1):34-42.

11. Budtz CR, Andersen NB, Qvist I, Pedersen F, Ladegourdie V, Ottosen J, et al. Monitoreringsprojekt fysioterapipraksis 2016: Kan en IT-baseret grundmodel for udredning, behandling og kommunikation implementeres $\mathrm{i}$ praksissektoren? [Project on monitoring physiotherapy practice 2016: can standardised electronic data collection tools be implemented ?]. https:// www.sundhed.dk/sundhedsfaglig/information-til-praksis/midtjylland/ fysioterapeut/rapporter-mm/monitoreringsprojekt/. Accessed 5 Nov 2018.

12. Kamper SJ, Maher CG, Mackay G. Global rating of change scales: a review of strengths and weaknesses and considerations for design. J Man Manip Ther. 2009;17(3):163-70

13. de Vet HC, Ostelo RW, Terwee CB, van der Roer N, Knol DL, Beckerman H, et al. Minimally important change determined by a visual method integrating an anchor-based and a distribution-based approach. Qual Life Res. 2007; 16(1):131-42.

14. Jorgensen $C K$, Fink P, Olesen F. Patients in general practice in Denmark referred to physiotherapists: a description of patient characteristics based on general health status, diagnoses, and sociodemographic characteristics. Phys Ther. 2001;81(3):915-23.

15. de Vos Andersen NB, Kent P, Hjort J, Christiansen DH. Clinical course and prognosis of musculoskeletal pain in patients referred for physiotherapy: does pain site matter? BMC Musculoskelet Disord. 2017;18(1):130 -017-14873.

16. Mokkink LB, Terwee CB, Knol DL, Stratford PW, Alonso J, Patrick DL, et al. The COSMIN checklist for evaluating the methodological quality of studies on measurement properties: a clarification of its content. BMC Med Res Methodol. 2010;10:22 2288-10-22.

17. Mintken PE, Glynn P, Cleland JA. Psychometric properties of the shortened disabilities of the arm, shoulder, and hand questionnaire (QuickDASH) and numeric pain rating scale in patients with shoulder pain. J Shoulder Elb Surg. 2009;18(6):920-6.

18. Franchignoni F, Vercelli S, Giordano A, Sartorio F, Bravini E, Ferriero G. Minimal clinically important difference of the disabilities of the arm, shoulder and hand outcome measure (DASH) and its shortened version (QuickDASH). J Orthop Sports Phys Ther. 2014;44(1):30-9.

19. Chester R, Jerosch-Herold C, Lewis J, Shepstone L. The SPADI and QuickDASH are similarly responsive in patients undergoing physical therapy for shoulder pain. J Orthop Sports Phys Ther. 2017:47(8):538-47.

20. Polson K, Reid D, McNair PJ, Larmer P. Responsiveness, minimal importance difference and minimal detectable change scores of the shortened disability arm shoulder hand (QuickDASH) questionnaire. Man Ther. 2010;15(4):404-7.

21. Ostelo RW, Deyo RA, Stratford P, Waddell G, Croft P, Von Korff M, et al. Interpreting change scores for pain and functional status in low back pain: towards international consensus regarding minimal important change. Spine (Phila Pa 1976). 2008;33(1):90-4.

22. Frahm Olsen M, Bjerre E, Hansen MD, Tendal B, Hilden J, Hrobjartsson A. Minimum clinically important differences in chronic pain vary considerably by baseline pain and methodological factors: systematic review of empirical studies. J Clin Epidemiol. 2018;101:87-106.e2.

23. Danish National Committee on Biomedical Research Ethics. Act on research ethics review of health research projects. 2013; Available at: http://www.nvk. dk/english/act-on-research. Accessed 05/03, 2018.

Ready to submit your research? Choose BMC and benefit from:

- fast, convenient online submission

- thorough peer review by experienced researchers in your field

- rapid publication on acceptance

- support for research data, including large and complex data types

- gold Open Access which fosters wider collaboration and increased citations

- maximum visibility for your research: over $100 \mathrm{M}$ website views per year

At BMC, research is always in progress.

Learn more biomedcentral.com/submissions 